University of Nebraska - Lincoln

DigitalCommons@University of Nebraska - Lincoln

Faculty Publications, Department of Psychology

Psychology, Department of

January 1996

\title{
A Cross-National Study on the Relations Among Prosocial Moral Reasoning, Gender Role Orientations, and Prosocial Behaviors
}

\author{
Gustavo Carlo \\ University of Nebraska-Lincoln, carlog@missouri.edu \\ Silvia H. Koller \\ Universidade Federal de Rio Grande do Sul, Porto Alegre, Brazil \\ Nancy Eisenberg \\ Arizona State University \\ Marcia S. Da Silva \\ Universidade Federal de Rio Grande do Sul, Porto Alegre, Brazil \\ Claudia B. Frohlich \\ Universidade Federal de Rio Grande do Sul, Porto Alegre, Brazil
}

Follow this and additional works at: https://digitalcommons.unl.edu/psychfacpub

Part of the Psychiatry and Psychology Commons

Carlo, Gustavo; Koller, Silvia H.; Eisenberg, Nancy; Da Silva, Marcia S.; and Frohlich, Claudia B., "A CrossNational Study on the Relations Among Prosocial Moral Reasoning, Gender Role Orientations, and Prosocial Behaviors" (1996). Faculty Publications, Department of Psychology. 296.

https://digitalcommons.unl.edu/psychfacpub/296

This Article is brought to you for free and open access by the Psychology, Department of at DigitalCommons@University of Nebraska - Lincoln. It has been accepted for inclusion in Faculty Publications, Department of Psychology by an authorized administrator of DigitalCommons@University of Nebraska - Lincoln. 


\title{
A Cross-National Study on the Relations Among Prosocial Moral Reasoning, Gender Role Orientations, and Prosocial Behaviors
}

\author{
Gustavo Carlo, Department of Psychology, University of Nebraska-Lincoln \\ Silvia H. Koller, Department of Psychology, Universidade Federal de Rio Grande do Sul, Porto Alegre, Brazil \\ Nancy Eisenberg, Department of Psychology, Arizona State University \\ Marcia S. Da Silva, Department of Psychology, Universidade Federal de Rio Grande do Sul, Porto Alegre, Brazil \\ Claudia B. Frohlich, Department of Psychology, Universidade Federal de Rio Grande do Sul, Porto Alegre, Brazil
}

\begin{abstract}
This research examined the correlates of prosocial moral reasoning (PMR) in 2 studies. Study I investigated age, gender, and culture group differences in PMR in Brazilian children and adolescents $(n=265)$ and U.S. adolescents $(n=$ 67). Relations between PMR and both prosocial behaviors and gender role orientations in Brazilian adolescents $(n=136)$ were explored in Study 2. Self-reflective, internalized reasoning was positively related, and hedonistic reasoning was negatively related, to peer ratings of prosocial behaviors. Femininity was associated with more self-reflective, internalized concerns and with less concerns regarding gaining others' approval. In general, age and gender differences in PMR were similar for both Brazilian and U.S. adolescents. However, U.S. adolescents scored higher on internalized moral reasoning than Brazilian adolescents. Discussion focused on the correlates of PMR in Brazilian and U.S. children.
\end{abstract}

Children and adolescents often are faced with the decision to help others at some cost to themselves. Many times, these decisions are difficult because they arise in situations in which formal external guidelines are absent or unclear. Decisions in those contexts have been the focus of researchers interested in prosocial moral reasoning (i.e., reasoning about moral dilemmas in which one person's needs or desires conflict with those of needy others in a context in which the role of prohibitions, authorities' dictates, and formal obligations are minimal or absent; Eisenberg, 1986; see Rest, 1983).

According to Eisenberg (1986), developmental changes in prosocial moral reasoning are somewhat consistent with developmental changes in justice-oriented, Kohlbergian (Colby, Kohlberg, Gibbs, \& Lieberman, 1983) moral reasoning. This similarity is due to the role of cognition as a necessary but not sufficient factor for some types of moral reasoning. However, Esienberg (Esienberg \& Shell, 1986); Eisenberg et al., 1987) has argued and presented some supportive evidence that individuals' level of prosocial moral reasoning, within the limits of their sociocognitive competence, varies as a function of personal (e.g., sympathetic tendencies) and contextual (e.g., cost of helping) factors. Following this argument, social contextual factors such as culture might be expected to influence level of prosocial moral reasoning depending on the values and socialization emphases in the culture.

We thank Richard Dienstbier, Janis Jacobs, and Ross Thompson for their helpful suggestions and Laassa Eschiletti, Sandra C. Palma, and Ana C. Svirski for their assistance. This project was partially supported by a Faculty Summer Research Fellowship from the Office of the Research Council.

Corresponding author: Gustavo Carlo, Department of Psychology, University of Nebraska-Lincoln, 219 Burnett Hall, Lincoln, Nebraska 68588-0308
Furthermore, because education and logical skills appear to be associated with level of moral reasoning (Colby et al., 1983; Eisenberg, 1986), differences in educational experiences may result in cross-cultural variations in prosocial moral reasoning (particularly at the highest levels).

Consistent with cognitive developmental theory, researchers frequently have found that the sophistication of moral judgment increases during adolescence, presumably due in part to an increase in perspective taking and reflective abstract cognitive skills (Colby et al., 1983; Eisenberg, 1986; Rest, 1983; Selman, 1980). In a series of longitudinal and cross-sectional studies on children and adolescents from the United States, Eisenberg and her colleagues have found several age-related changes in several types of prosocial moral reasoning (Eisenberg, Carlo, Murphy, \& Van Court, 1995; Eisenberg, Miller, Shell, McNalley, \& Shea, 1991). For example, when an interview measure of prosocial moral reasoning was used, judgments regarding gaining the approval of others and global, stereotyped notions about good or bad behaviors tended to increase in adolescence until between the ages of 13-14 and 15-16 years, and then decreased somewhat in frequency. In contrast, self-reflective perspective taking and internalized norms judgments tend to emerge in late childhood and increase through adolescence (Eisenberg et al., 1991; Eisenberg et al., 1995). In addition, researchers interested in the motives for prosocial behaviors have presented a pattern of findings that is similar and consistent with the aforementioned pattern. For example, intrinsic (e.g., internalized or other-oriented) motives for prosocial behavior appear to be relatively high during adolescence in industrialized European societies such as Germany, Italy, and Poland (Boehnke, Silbereisen, Eisenberg, Reykowski, \& Palmonari, 
1989), as well as in other countries such as Israel (Bar-Tal, Raviv, \& Lewis-Levin, 1980; Raviv, Bar-Tal, \& Lewis-Levin, 1980).

At present, there is relatively little cross-cultural research on prosocial moral reasoning. Nonetheless, some research suggests that the prosocial moral reasoning of elementary school children and adolescents is similar (although relatively small differences have been found) across various industrialized societies (Eisenberg, 1986; Eisenberg, Boehnke, Schuler, \& Silbereisen, 1985). For example, Israeli kibbutz children expressed more concern with the humaneness of recipients and internalized norms and laws than Anglo-Americans or Israeli city children (Fuchs, Eisenberg, Hertz-Lazarowitz, \& Sharabany, 1986). Furthermore, in one study on prosocial moral reasoning in a non-Western, non industrialized culture, researchers found that adolescents from two Maisan coastal communities in Papua New Guinea verbalized virtually no higher-level internalized, stereotypic, or sympathetic moral reasoning (Eisenberg, 1986), although such reasoning is found in adolescents from Western and industrialized communities. Instead, the Papua New Guinea adolescents used substantial amounts of reasoning based on the needs of others, concern with assisting others connected or liked by one's self, and pragmatic concerns. These types of reasoning presumably reflect the personal ties and interactions and the collectivist orientation of their society (Tietjen, 1986). In brief, prosocial moral reasoning appears to be similar in the few industrialized societies examined thus far. However, cultural factors do seem to influence the frequency and report of some types of reasoning across Western and non-Western societies.

Brazilians, particularly from the southern region, in many respects are perhaps the most Westernized people in Latin America (Poppino, 1973). ${ }^{1}$ The southern region of Brazil is the country's most populous region and is a center for manufacturing, agriculture, and technology. In this region, approximately $80 \%$ of the workforce earns more than the region's minimum wage, infant mortality rates are about a third lower than in the northeast region of Brazil, and most children stay in school at least through the fourth grade (Lang, 1988). According to Hofstede (1982), on the whole, Brazil ranks close to the United States on the masculinity-femininity dimension (i.e., relative importance of advancement, earnings, and recognition). In addition, some researchers (Haidt, Koller, \& Dias, 1993) have found that judgment of mores about specific affect-laden issues generally were comparable between individuals from this region of Brazil and a sample of individuals from the United States; social mores varied more as a function of socioeconomic status than culture. Nonetheless, there is evidence that Brazilian society is oriented toward collectivism (i.e., less personal time and freedom, more dependency on a system) and personal and interpersonal relationships to a greater degree than the majority of society in the United States (Botenmpo, Lobel, \& Triandis, 1990; Hofstede, 1982).

An emphasis on collectivism and interpersonal relationships in Brazilian society might be reflected in some modes of prosocial moral reasoning, including generalized reciprocity reasoning (i.e., the belief that helping would benefit everyone) or affectional relationship reasoning (i.e., consideration of the existing relationship with the needy individual). Presumably, some socialization practices in Brazil would be aimed at promoting and nurturing collectivism and personal ties and contact. Consistent with this notion, Biaggio (1976) found that Brazilians scored high on
Kohlbergian Stage 3 (i.e., concern with interpersonal relationships) moral reasoning. However, note that generalized reciprocity reasoning, in contrast to Stage 3 in Kohlberg's moral reasoning scheme, is a high-level mode of prosocial moral reasoning. Moreover, there is some evidence (Botenmpo et al., 1990) that Brazilians (from Rio De Janeiro) may behave prosocially without much concern for self-presentation (i.e., they appear to be intrinsically motivated). In summary, we know little concerning the preferred modes of prosocial moral reasoning in Brazilians when compared directly to individuals from the United States. Consequently, it is difficult to predict whether generalized reciprocity and other high-level modes of prosocial moral reasoning would be more preferred by Brazilians or individuals from the United States.

Gender is another social category that has been linked to individual differences in prosocial moral reasoning. This issue is important in order to address claims (e.g., Gilligan, 1982) that there are cultural biases in moral reasoning. To date, however, the answer to this question remains relatively unclear (see Baumrind, 1986; Gilligan \& Attanucci, 1988; Eisenberg, Fabes, \& Shea, 1989; Walker, 1991). Eisenberg(Eisenberg et al., 1989) and others (Gilligan, 1982) have theorized that gender-specific socialization practices may lead to gender differences in care-oriented modes of moral reasoning. Indeed, in adolescence, girls sometimes have expressed more higher level, other-oriented modes of prosocial moral reasoning (e.g., perspective taking and internalized affect about consequences-type reasoning; Eisenberg et al., 1995), although this pattern seemed stronger in early rather than late adolescence (see Eisenberg et al., 1991; Eisenberg et al., 1995). Furthermore, adolescent girls have exhibited somewhat higher moral reasoning overall than have adolescent boys (Eisenberg et al., 1989; Eisenberg et al., 1995).

In research with a paper-and-pencil measure of prosocial moral reasoning, some gender differences also have been found. For example, girls from the United States score higher on internalized moral reasoning and lower on approval-oriented than do boys (Eisenberg et al., 1995) and also score higher than boys on stereotypic reasoning (Carlo, Eisenberg, \& Knight, 1992). Because of the similarities between Anglo-American and Brazilian societies in gender roles (Hofstede, 1982), we anticipated that Brazilian adolescents might display gender differences similar to those in the U.S. sample.

To date, most of the research on prosocial moral reasoning has been conducted using interview measures of moral reasoning. However, Carlo et al. (1992) recently introduced a paper-andpencil measure of prosocial moral reasoning (the prosocial reasoning objective measure, or PROM) designed to examine prosocial moral reasoning in adolescence. As pointed out by these and other researchers (e.g., Gibbs et al., 1984; Kurtines \& Pimm, 1983; Rest, 1983), paper-and-pencil measures of moral reasoning have been designed to assess the individual's ability to choose among alternative moral viewpoints, a skill that is related, but distinct, from spontaneously producing, elaborating, and defending moral viewpoints (as in interview measures). Paper-and-pencil

\footnotetext{
${ }^{1}$ Some authors have raised concerns on the use of global, stereotypic terms (e.g., collectivism and Westernized) to describe culture. These terms were used with the knowledge that there are wide variations on psychological variables within cultures.
} 
measures facilitate verification of standardized administration, enhance comparability of findings across studies, and may require less verbal skills than interview measures. The benefits of paperand-pencil measures may be particularly evident when conducting studies in different countries and in applied settings.

In summary, based on social and economic similarities between specific regions of Brazil and the United States and on the cognitive prerequisites for prosocial moral reasoning, we hypothesized that the pattern of age-related changes in prosocial moral reasoning for Brazilian children and adolescents would be similar to that of U.S. children and adolescents. Furthermore, girls were expected to score higher on other-oriented and internalized modes of prosocial moral reasoning (although no cultural differences in the pattern of gender differences were expected). In the first part of Study 1, the age- and gender-related patterns of prosocial moral reasoning in Brazilian children and adolescents were examined. In the second part, the moral reasoning of Brazilian adolescents (aged 11 to 15 years) was compared with the moral reasoning of a similar age group of Anglo-American adolescents (selected from the sample used in Carlo et al., 1992).

\section{Study 1: Part 1}

\section{Method}

\section{Participants}

The participants were 271 fifth- through tenth-grade children and adolescents (128 male, 143 female) from private schools in a predominantly White, middle-class community in a southern city (Porto Alegre) of Brazil. Six students left some items blank on the questionnaire and were dropped from the main analyses. Thus, there were 265 students (127 male, 138 female; $M$ age $=14.6$ years, $S D=1.9$ years) in the final sample. Participation in the study was voluntary, and the students received no material compensation for participating.

\section{Materials}

The paper-and-pencil measure of prosocial moral reasoning (Carlo et al., 1992) was based on a previously developed (see Eisenberg et al., 1987) interview measure of prosocial moral reasoning. The PROM was translated into Portuguese and back into English by a researcher who is fluent in both Portuguese and English and who is an expert in moral development (Silvia H. Koller). Then the PROM was translated back into Portuguese, and the translation was confirmed by a fellow researcher who is fluent in both English and Portuguese. The PROM contained seven story dilemmas designed to invoke a conflict between the actor's needs, wants, and desires and those of another (or others). The dilemmas dealt with the following issues: (a) choosing to get an injured child's parents versus going to a friend's party, (b) keeping food after a flood versus giving some food to others who had none, (c) helping disabled children strengthen their legs by teaching them to swim versus practicing for a swimming contest to win prize money, (d) continuing to stay and play in one's own backyard versus going to try and stop a bully that is picking on a peer, (e) going to the beach with friends versus helping a peer to study for and pass a math test, (f) donating blood to a needy other versus losing time and money at work and school, and (g) helping a peer who is being teased versus risking rejection from peers.

The following is a sample story from the PROM (English version):

One day Mary was going to a friend's party. On the way, she saw a girl who had fallen down and hurt her leg. The girl asked Mary to go to the girl's house and get her parents so the parents could come and take her to a doctor. But if Mary did run and get the girl's parents, Mary would be late to the party and miss the fun and social activities with her friends.

The order of the PROM stories was randomized for each student, the protagonists were the same gender as the student, and there was a practice story at the beginning. After reading each story, adolescents were first asked to indicate whether (a) the protagonist should help the needy other, (b) the protagonist should not help the needy other, or (c) they were unsure what the protagonist should do. Following this decision, the students were asked to rate the importance of six considerations pertaining to why the protagonist should or should not help the needy other in the story (on a 5-point scale; $1=$ not at all, 5 = greatly). The PROM took each student about 15 to $20 \mathrm{~min}$ to complete.

A representative sampling of frequently reported prosocial moral reasoning choices was selected for each story. Each of the stories (there were seven stories) included one hedonistic item (Level 1 in Eisenberg's, 1986, schema, which included simple hedonistic or direct reciprocity reasoning; e.g., "It depends how much fun Mary expects the party to be and what sorts of things are happening at the party"; Cronbach's $\alpha=.61$ ), one needs-oriented item (Level 2; e.g., "It depends whether the girl really needs help or not"; $\alpha=.60)$, one approval-oriented item (Level 3; e.g., "It depends whether Mary's parents and friends will think she did the right or she did the wrong thing"; $\alpha=.85$ ), and one stereotypic item (Level 3; e.g., "It depends if Mary thinks it's the decent thing to do or not"; $\alpha=.61$ ). In addition, each of the stories contained one item that reflected higher level reasoning (Levels 4 and 5; i.e., sympathetic, perspective taking, internalized affect, or abstract internalized reasoning; e.g., "It depends how Mary would feel about herself if she helped or not"; $\alpha=.61$ ). The sixth reasoning choice was a lie/nonsense item (e.g., "It depends whether Mary believes in people's values of metacognition or not"). ${ }^{2}$

\section{Procedure}

All adolescents were administered a demographic questionnaire and the PROM. The session lasted about 20 to $30 \mathrm{~min}$ and was conducted in the classrooms (maximum group size of 30). Students then were carefully debriefed and thanked.

\section{Scoring of the PROM}

For each participant, PROM ratings that corresponded to one of the five types of prosocial moral reasoning were summed across the seven stories to obtain a frequency score. A frequency score also was obtained using the lie/nonsense items in the PROM; however, this scale was used only to assess whether students scored 2 standard deviations or higher on this scale (as suggested by Carlo et al., 1992), and no adolescents met this criterion.

As in prior studies (Carlo et al., 1992; Eisenberg et al., 1995), preliminary analyses using the frequency PROM scores suggested that there was a response bias in the use of the scale. Students tended to use either the lower or the higher ends of the PROM scale. Thus, the frequency PROM scores were transformed to proportion PROM scores (see Boehnke et al., 1989, for a similar procedure) by dividing each of the PROM scale scores (reflecting the five types of reasoning) by the sum of the PROM scale scores. Conceptually, the proportion scores reflect a participant's preference for a reasoning type

\footnotetext{
${ }^{2}$ Both the English and Portugese versions of the PROM may be obtained from Gustavo Carlo on request.
} 
Table 1. Means and Standard Deviations for Prosocial Moral Reasoning by Samples in Study 1

\begin{tabular}{|c|c|c|c|c|c|c|c|c|}
\hline \multirow[b]{3}{*}{ Moral reasoning } & \multirow{2}{*}{\multicolumn{2}{|c|}{$\begin{array}{c}\text { Part } 1 \\
(N=265)\end{array}$}} & \multicolumn{6}{|c|}{ Part 2} \\
\hline & & & \multicolumn{2}{|c|}{$\begin{array}{c}\text { Brazil } \\
(n=211)\end{array}$} & \multicolumn{2}{|c|}{$\begin{array}{l}\text { United States } \\
\quad(n=63)\end{array}$} & \multicolumn{2}{|c|}{$\begin{array}{c}\text { Total } \\
(N=174)\end{array}$} \\
\hline & $M$ & $S D$ & $M$ & $S D$ & $M$ & $S D$ & $M$ & $S D$ \\
\hline Hedonistic & .18 & .03 & .18 & .03 & .17 & .04 & .18 & .03 \\
\hline Approval & .15 & .05 & .15 & .05 & .14 & .05 & .15 & .05 \\
\hline Needs-oriented & .21 & .03 & .23 & .04 & .22 & .04 & .21 & .03 \\
\hline Stereotyped & .23 & .04 & .21 & .03 & .21 & .04 & .23 & .04 \\
\hline Internalized & .24 & .03 & .24 & .03 & .26 & .04 & .24 & .04 \\
\hline
\end{tabular}

Note. The Brazilian sample in Part 2 is a subsample of the Brazilian sample in Part 1 selected to match the Anglo-American sample on age range.

in relation to the other reasoning types. In the subsequent analyses of the PROM, proportion scores were used rather than frequency scores.

\section{Results}

Table 1 presents the means and standard deviations for the PROM scales for Part 1. A series of hierarchical regression analyses using prosocial moral reasoning scores as the criterion were conducted to assess age and gender differences in prosocial moral reasoning. As suggested by Aiken and West (1991), all predictors were centered (by subtracting the means) before the analyses to reduce nonessential multicollinearity. In each of the analysis (there were five analyses corresponding to the five prosocial moral reasoning scales), age and gender were entered on the first block and then the Age $\times$ Gender interaction vector was entered on the second block.

There were significant main effects of both age and gender on the first step for approval-oriented, stereotypic, and internalized moral reasoning, $F \mathrm{~s}(2,262)=19.90,12.44$, and 6.51 , $p<.001, p<.001$, and $p<.005\left(R^{2}\right.$ changes $=.13, .09$, and $.05)$, respectively. Younger children and boys scored higher on approval-oriented reasoning, $t \mathrm{~s}(262)=-4.31$ and $-4.67, p<$ .001 (standardized $B \mathrm{~s}=-.25$ and -.27 ). In contrast, children scored higher on stereotypic and internalized reasoning as age increased, $t \mathrm{~s}(262)=3.05$ and $2.69, p<.005$ and $p<.01$ (standardized $B \mathrm{~s}=.18$ and .16 ), respectively, and girls scored higher than boys on both stereotypic and internalized reasoning, $t$ $\mathrm{s}(262)=4.00$ and $2.44, p \mathrm{~s}<.001$ and .02 (standardized $B \mathrm{~s}=$ .24 and .15), respectively. There were no other significant main or interaction effects.

We conducted an additional set of analyses to examine linear and quadratic trends in prosocial moral reasoning because some types of reasoning have been found to show both decreases and increases in usage during childhood and adolescence (Eisenberg et al., 1995). In these analyses, there was a significant Quadratic Trend $\times$ Gender interaction for hedonistic moral reasoning, $F(5,259)=3.86, p<.05\left(R^{2}\right.$ change $=$ $.02)$; however, tests of the simple trends for each gender were not significant. There were no other significant quadratic trend main or interaction effects. ${ }^{3}$

\section{Study 1: Part 2}

The second part of Study 1 was designed to directly compare age, gender, and cultural group differences in Brazilian and Anglo-American adolescents' ratings of prosocial moral reasoning.

\section{Method}

\section{Participants}

The Brazilian participants were 219 students (a subset of the sample from Study 1) matched to the U.S. sample by age (selected if age range was from 141 to 198 months; 106 male, 113 female; $M$ age = 14.2 years, $S D=1.5$ years).

The students from the United States were 67 adolescent students ( 25 male, 42 female) from a prior published study on prosocial moral reasoning (Carlo et al., 1992). The U.S. students were selected only if they were between 141 months and 198 months of age $(2$ students from the original sample were dropped on the basis of this criterion; for the final sample, $M$ age $=14.2$ years, $S D=1.7$ years). Four students were dropped from some analyses because they left some items on the PROM unanswered. The students were predominantly White, middleclass, and from public junior high or high schools in Tempe, Arizona. The students received $\$ 10$ ( 2 tenth graders received $\$ 20$ in an effort to recruit more male students) to motivate voluntary participation.

\section{Materials}

The paper-and-pencil measure of prosocial moral reasoning (PROM; Carlo et al., 1992) was the same as described earlier. Although the Brazilians were administered all seven stories from the PROM (see Part 1), only the same five stories (the first five stories described earlier) that were administered to the U.S. sample were used for analyses. The scoring procedures were the same as described previously. Two Brazilian boys scored 2 standard deviations above the mean on the lie/nonsense scale of the PROM and were dropped from subsequent analyses.

\section{Procedure}

As mentioned earlier, the Brazilian adolescents participated in an experimental session in which they completed a demographic infor-

\footnotetext{
${ }^{3}$ Significant nonlinear trend interactions are a special case of moderators and are often difficult to detect (McClelland \& Judd, 1993).
} 
mation sheet and the PROM questionnaire. The session lasted about $30 \mathrm{~min}$ and was conducted in the classrooms (maximum group size of 30). The U.S. adolescents participated in two experimental sessions (see Carlo et al., 1992), but only the demographic information and PROM questionnaire data from the first session were used in the present study. In this session, the demographic information and the PROM were administered before all other questionnaires were administered. In both the Brazilian and Anglo-American sessions, participating students were carefully debriefed and thanked.

\section{Results}

Table 1 presents the means and standard deviations for the PROM scales for the Brazilian sample, the U.S. sample, and the total sample in Part 2. A series of hierarchical regression analyses using prosocial moral reasoning scores as the criterion was conducted to assess age, gender, and group differences in prosocial moral reasoning (see comparable analyses above). In each analysis, age (in months), gender, and culture group were entered in the first block; then all possible two-way interaction vectors were entered in the second block; lastly the Age $\times$ Gender $\times$ Culture Group interaction vector was entered in the third block.

There were significant main effects of age and culture group on the first step for internalized reasoning, $F(3,270)=8.00, p<$ $.001\left(R_{2}\right.$ change $\left.=.08\right)$. Older children and U.S. children scored higher on internalized reasoning, $t \mathrm{~s}(270)=2.71$ and 3.51, $p \mathrm{~s}<$ .01 and .001 (standardized $B \mathrm{~s}=.16$ and .21 ), respectively.

There also were significant main effects of age and gender on the first step for approval-oriented and stereotypic moral reasoning, $F \mathrm{~s}(3,270)=13.81$ and 7.43 , both $p \mathrm{~s}<.001\left(R_{2}\right.$ change $=.13$ and .08), respectively. Younger children and boys scored higher on approval-oriented reasoning, $t \mathrm{~s}(270)=-4.08$ and $-4.75, p \mathrm{~s}<.001$ (standardized $B \mathrm{~s}=-.23$ and -.27 ), respectively. In contrast, older children and girls scored higher on stereotypic reasoning, $t \mathrm{~s}(270)=2.56$ and 3.77, $p \mathrm{~s}<.01$ and .001 (standardized $B \mathrm{~s}=.15$ and .22 ), respectively. There were no other significant main effects, and there were no significant two- or three-way interaction effects.

\section{Discussion of Study 1}

The present findings from both parts of Study 1 were generally consistent with the hypotheses and yielded several interesting findings. More specifically, the age, gender, and cultural group differences were generally consistent with prior findings from studies using children and adolescents from Western and non-Western, industrialized countries.

Of particular interest was the one cultural group difference on prosocial moral reasoning in Part 2. Children from the United States scored higher on internalized moral reasoning compared with Brazilian children. This finding is somewhat consistent with other researchers' findings that Brazilians score approximately one stage lower than Americans on Kohlbergian moral reasoning (Hutz, De Conti, \& Vargas, 1993). Because moral reasoning has been found to be significantly related to education and logical skills (Colby et al., 1983; Eisenberg, 1986), it may be that the culture difference was partly a function of these variables. Although children from both samples were recruited from schools, there may be differences in the degree to which the educational systems stress critical reasoning skills that may be necessary for developmentally sophisticated moral reasoning.

Despite the moderate sample size in this study, there were no differences between U.S. and Brazilian adolescents in age or gender effects on prosocial moral reasoning (i.e., there were no significant interactions of age or gender with cultural group in Part 2). Indeed, consistent with Eisenberg et al.'s (1995) findings, approval-oriented reasoning declined with age whereas internalized reasoning increased with age (in Carlo et al., 1992, internalized reasoning increased with age particularly for male participants). In addition, stereotypic reasoning increased with age in both studies. This latter finding may not be surprising given that stereotypic reasoning appears to increase with age sometime in mid-adolescence (Eisenberg et al., 1991) before declining in frequency.

With regard to gender differences in prosocial moral reasoning, there were several findings of interest. Girls preferred stereotypic reasoning and rejected approval-oriented reasoning more than did boys. The former finding was consistent with the suggestion that girls, compared with boys, may be more exposed to global, traitlike verbalizations in socialization opportunities (Carlo et al., 1992). It was less clear why girls did not report more internalized moral reasoning than boys in Part 2 of Study 1 as in the first part and as in prior studies using the PROM (Eisenberg et al., 1995).

\section{Study 2}

As might be expected, prosocial moral reasoning has been theoretically and empirically linked to prosocial behaviors (Eisenberg, 1986; Underwood \& Moore, 1982). The higher levels of prosocial moral reasoning often reflect other-oriented concerns with the needs of distressed individuals and are consonant with helpfulness and generosity toward others. Moreover, cognitively sophisticated individuals may be more apt to understand and consider the relevant situational and personal factors necessary for engaging in helping behaviors (Carlo, Knight, Eisenberg, \& Rotenberg, 1991).

Eisenberg and her colleagues (Eisenberg \& Shell, 1986; Eisenberg et al., 1987; Eisenberg et al., 1991; Eisenberg et al., 1995) have found in general that prosocial behaviors are negatively associated with hedonistic modes of moral reasoning and positively associated with needs-oriented moral reasoning and a composite index of moral reasoning. In addition, in one study (Eisenberg et al., 1995), using a paper-and-pencil measure of prosocial moral reasoning (the PROM), researchers found a positive relation between a prosocial behavior aggregate and internalized moral reasoning and a moral reasoning composite, and a negative relation between the prosocial behavior aggregate and hedonistic moral reasoning. In contrast, self-oriented, hedonistic modes of reasoning have been negatively associated with prosocial behaviors. The present study was designed to extend the prior findings by examining these relations in adolescents from a country (i.e., Brazil) other than the United States.

To our knowledge, one published study (Eisenberg et al., 1985) was designed to investigate the relations between prosocial moral reasoning and prosocial behaviors in a country other than the United States. In this study, German children who donated 
candies were likely to report needs-oriented moral reasoning and scored relatively high on a prosocial moral reasoning composite, and they were less likely to report hedonistic moral reasoning. In addition, picking up paper clips was not significantly related to prosocial moral reasoning. In an unpublished study (Aykel \& Aykel, 1991), researchers found a significant positive relation between a composite of prosocial moral reasoning (using Eisenberg's, 1986, scheme) and a prosocial behavior composite using Turkish high school students. Furthermore, these latter researchers found a significant negative relation between hedonistic reasoning and the prosocial behavior composite and a significant positive relation between internalized reasoning and the prosocial behavior composite. In two studies on Israeli children's motives for prosocial behaviors, researchers (Bar-Tal et al., 1980; Raviv et al., 1980) found that children's justifications for helping others varied and included egoistic (some direct reciprocity), normative (following a social norm or expectation), and altruistic motives. However, in general, those children who spontaneously donated most were motivated primarily by higher level altruism (i.e., generalized reciprocity motives and as a result of moral convictions and without expectations of external rewards). In summary, the findings regarding the relations between prosocial moral reasoning or motives and prosocial behaviors in German, Israeli, and Turkish children generally have been consistent with findings reported in studies using children from the United States.

Based on the argument that the influences of prosocial behaviors in Brazilian adolescents would be similar to those in adolescents from the United States and on the generally consistent prior findings in cultures other than the United States, higher levels of moral reasoning (e.g., internalized) were expected to be positively related to prosocial behaviors. In contrast, self-focused, hedonistic prosocial moral reasoning was expected to be negatively related to prosocial behaviors (unless there was some expectation of direct reciprocity from helping).

As mentioned previously, another issue of interest to researchers is whether moral reasoning in male and female individuals differs (e.g., Walker, 1991). A somewhat different approach to address this issue is to examine whether specific forms of moral reasoning are associated with masculine or feminine role characteristics that are often considered culture-based (McGraw \& Bloomfield, 1987). Based on the assumption that adolescents have clear stereotyped conceptions of gender roles, it may be that specific moral justifications are perceived as specific to a masculine or feminine orientation. It is these stereotyped conceptions that may, in turn, influence adolescents' reports of specific types of moral reasoning.

The study of gender role orientations in prosocial moral reasoning would be of particular interest to moral development researchers because prosocial moral reasoning is oriented toward caring and interpersonal relationships (Eisenberg, 1986), which are commonly feminine-typed concerns (Gilligan, 1982). Whereas there is little empirical support for the notion that gender role orientation and moral reasoning are significantly related with regard to Kohlberg's (Colby et al., 1983) justice-oriented moral reasoning approach (McGraw \& Bloomfield, 1987), there is evidence that there are gender differences in some types of prosocial moral reasoning (at least in early adolescence; Eisenberg et al., 1989). For example, by adolescence, needs-oriented, empathic, role-taking, and internalized prosocial moral reason- ing sometimes are preferred by girls compared with boys. However, the relations between gender role orientations and prosocial moral reasoning have not been directly examined.

In brief, there is evidence that female adolescents prefer some types of internalized reasoning more than do male adolescents (Carlo et al., 1992; Eisenberg et al., 1989); that some higher levels of prosocial moral reasoning include feminine-typed, emotionally based modes of reasoning forms (e.g., empathic reasoning); and that Brazilian and U.S. societies do not appear to differ in emphasizing masculinity-femininity (Hofstede, 1982). Thus, femininity was expected to be positively related to higher level prosocial moral reasoning (e.g., internalized) and negatively related to lower level prosocial moral reasoning (e.g., approval-oriented, hedonistic). Masculinity was expected to be unrelated to prosocial moral reasoning.

\section{Method}

\section{Participants}

Participants were 136 adolescents (57 male, 79 female) from private school classrooms in a southern city (Porto Alegre) of Brazil. The students were mostly White Brazilians recruited from a predominantly middle-class neighborhood. Of these adolescents, 6 did not complete one of the scales (the Sex Role Inventory) and were dropped from the main analyses (although their data are included in the preliminary analyses). Thus, there were 130 adolescents (55 male, $M$ age $=14.8$ years, $S D=1.5$ years; 75 female, $M$ age $=15.1$ years, $S D=1.6$ years) in the final sample. The students received no material compensation for participation.

\section{Materials}

The measure of prosocial moral reasoning. The measure of prosocial moral reasoning (PROM; Carlo et al., 1992) that was used in the first study (see Part 1 of Study 1) was used in this study. As in Study 1, scores from the five prosocial moral reasoning scales were summed and then transformed to proportion scores. No students scored higher than 2 standard deviations above the mean on the lie/ nonsense scale. Cronbach's alphas for the seven-item scales were $.60, .85, .66, .71$, and .64 for hedonistic, approval-oriented, needsoriented, stereotypic, and internalized moral reasoning, respectively.

Sex Role Inventory. An adaptation (Hutz \& Koller, 1992) of Bem's (Bem, 1974) 60 adjective-item sex role orientation (Bem's Sex Role Inventory; BSRI) scale for use with Brazilians was used. The scale is composed of several subscales, including a Masculinity scale (present study's $\alpha=.80,20$ items) and a Femininity scale ( $\alpha$ $=.93,20$ items $)$. As expected, preliminary correlational analyses revealed that masculinity and femininity were not significantly interrelated, $r(130)=-.05, n s .{ }^{4}$

Peer ratings of prosocial behavior. Students were asked to name their three best friends from their class. Then the students were asked to rate each of their classmates' helpfulness and generosity (each were 7 -point scales, 1 = never, 7 = almost always). Scores were summed and divided by the number of ratings for each student to obtain a score for helpfulness and generosity. Correlational analysis revealed a significant positive relation between Helpfulness and

${ }^{4}$ Conceptually, femininity and masculinity were the constructs of interest, and several theorists (e.g., Pedhazur \& Tetenbaum, 1979; Taylor \& Hall, 1982) have raised serious concerns regarding the androgyny construct. Thus, only masculinity and femininity traits were used. 
Generosity scale scores, $r(136)=.67, p<.001$. Thus, a prosocial behavior composite was computed by summing the Helpfulness and Generosity scale scores, and this composite was used in the subsequent analyses.

\section{Procedure}

All adolescents participated in two experimental sessions that lasted about 30 to $45 \mathrm{~min}$ in their classrooms (each session had a maximum of 30 students). In the first session, the students were administered the BSRI first; then the students were asked to rate their friends' and fellow classmates' dispositions to help others and to be giving. About a week and a half later, the students were administered the PROM. All students then were carefully debriefed and thanked.

\section{Results}

\section{Tests of Sex Differences in PROM Scores, Masculinity, and Femininity}

Means and standard deviations on prosocial moral reasoning are presented in Table 2. A series of univariate analyses were computed to assess gender differences in prosocial moral reasoning, masculinity, and femininity (multivariate analyses could not be computed because the PROM scores were multicollinear). There was a significant effect of gender on approval-oriented reasoning, $F(1,134)=4.37, p<.05$. Boys scored higher than girls on approval-oriented reasoning (see Table 2). There was a significant effect of gender on femininity, $F(1,128)=78.62, p<.001$. As expected, girls scored higher than boys: $M$ for girls $=5.48, S D$ $=0.85$, and $M$ for boys $=4.02, S D=1.03$. There were no other significant effects. ${ }^{5}$

\section{Relations Between Prosocial Moral Reasoning and Prosocial Behavior Ratings}

We conducted a series of hierarchical multiple regression analyses to test homogeneity of slopes assumptions using the prosocial behavior ratings as the criterion variable. In these analyses, gender and prosocial moral reasoning (hedonistic, approval-oriented, needs-oriented, stereotyped, or internalized) were entered simultaneously in the first block, and then the Gender $\times$ Prosocial Moral Reasoning interaction was entered in the second block. There were two significant interaction effects.

Table 2. Means and Standard Deviations for Prosocial Moral Reasoning as a Function of Gender in Study 2

\begin{tabular}{|c|c|c|c|c|c|c|}
\hline \multirow[b]{3}{*}{ Moral reasoning } & \multirow{2}{*}{\multicolumn{2}{|c|}{$\begin{array}{c}\text { Overall } \\
(n=136)\end{array}$}} & \multicolumn{4}{|c|}{ Gender } \\
\hline & & & \multicolumn{2}{|c|}{$\begin{array}{l}\text { Male } \\
(n=57) \\
\end{array}$} & \multicolumn{2}{|c|}{$\begin{array}{l}\text { Female } \\
(n=79) \\
\end{array}$} \\
\hline & $M$ & $S D$ & $M$ & $S D$ & $M$ & $S D$ \\
\hline Hedonistic & .18 & .03 & .18 & .03 & .18 & .03 \\
\hline Approval & .13 & .04 & .14 & .04 & .13 & .04 \\
\hline Needs-oriented & .20 & .03 & .20 & .03 & .20 & .03 \\
\hline Stereotyped & .24 & .03 & .23 & .04 & .24 & .03 \\
\hline Internalized & .25 & .03 & .25 & .04 & .26 & .03 \\
\hline
\end{tabular}

Table 3. Correlations Between Prosocial Moral Reasoning and Prosocial Behavior Within and Across Gender in Study 2

\begin{tabular}{llcc}
\hline & \multicolumn{3}{c}{ Prosocial behavior } \\
\cline { 2 - 4 } Moral reasoning & Male & Female & Total \\
\hline Hedonistic & $-.24^{*}$ & -.16 & $-.20^{* *}$ \\
Approval & $-.40^{* * *}$ & -.01 & $-^{\mathrm{a}}$ \\
Needs-oriented & $.29^{* *}$ & .14 & $--^{\mathrm{b}}$ \\
Stereotyped & .13 & -.01 & .07 \\
Internalized & $.26^{*}$ & $.28^{* * *}$ & $.27^{* * *}$ \\
\hline
\end{tabular}

Note: $n=55$ for male and $n=75$ for female adolescents.

${ }^{a}$ Gender $\times$ Approval-Oriented Moral Reasoning interaction was significant

${ }^{\mathrm{b}}$ Gender $\times$ Needs-Oriented Moral Reasoning interaction was significant.

$* p<.10$, two-tailed. $* * p<.05$, two-tailed. $* * * p<.01$, two-tailed.

There was a significant Gender $\times$ Approval-Oriented Moral Reasoning interaction on prosocial behavior, $R^{2}$ change $=.04$ (over and above the contributions of the main effect vectors), $F(1,126)$ for $R^{2}$ change $=5.85, p<.05$ (multiple $R=.27$ ). For boys, approval-oriented reasoning was significantly negatively related to prosocial behavior (see Table 3 ). In addition, the Gender $\times$ Needs-Oriented Moral Reasoning interaction significantly predicted prosocial behavior, $R^{2}$ change $=.05, F(1,126)$ for $R^{2}$ change $=6.06, p<.05$ (multiple $R=.22$ ). For boys, needs-oriented reasoning was positively related to prosocial behavior (see Table 3). There were no other significant interaction effects.

We also computed zero-order correlations to assess the relations between the PROM and the prosocial behavior ratings across gender (all tests were two-tailed; see Table 3). As expected, internalized reasoning was significantly positively related to prosocial behavior. In contrast, hedonistic reasoning was significantly negatively related to prosocial behavior. There were no other significant relations. ${ }^{6}$

\section{Relations Between Gender Role Orientations and Prosocial Moral Reasoning}

We conducted two sets of hierarchical multiple regression analyses to test homogeneity of slopes assumptions using prosocial moral reasoning scores as the criterion variables. In these analyses, gender and gender role orientation (either masculinity or femininity) were entered simultaneously in the first block, and then the Gender $\times$ Gender Role Orientation interaction was entered in the second block. There were two significant interaction effects (see Table 4 )

\footnotetext{
${ }^{5}$ Correlational analyses on the relations between age and PROM, masculinity, and femininity also revealed that younger adolescents scored higher than older adolescents on approval-oriented reasoning, $r(130)=-.22, p<$ .01 . There were no other significant effects.

${ }^{6}$ Correlational analyses both across and within each gender revealed no significant relations between gender role orientations and prosocial behaviors. Results of the regression analyses were virtually identical to those presented when age (in months) was inserted in the first block, suggesting that age did not account for the relations between prosocial moral reasoning and prosocial behaviors.
} 
The Gender $\times$ Femininity Orientation interaction significantly predicted hedonistic reasoning, $R^{2}$ change $=.04$ (over and above the contributions of the main effect vectors), $F(1,126)$ for $R^{2}$ change $=5.66, p<.05$ (multiple $R=.22$. For boys, femininity was significantly, positively related to hedonistic reasoning (see Table 4). In addition, the Gender $\times$ Masculinity Orientation interaction significantly predicted approval-oriented reasoning, $R^{2}$ change $=.04, F(1,126)$ for $R^{2}$ change $=5.66, p<.05$ (multiple $R$ $=.22$ ). For boys, masculinity was negatively related to approvaloriented reasoning (see Table 4).

To examine the relations between gender role orientations and prosocial moral reasoning across gender, we conducted zero-order correlational analyses (all tests were two-tailed; see Table 4). As can be seen in the table, femininity was negatively related to approval-oriented reasoning and positively related to internalized reasoning. There were no other significant relations.

\section{Discussion of Study 2}

In general, the present findings were consistent with the hypotheses and provide additional information about the correlates of adolescents' prosocial moral reasoning. As expected, several subscales of the paper-and-pencil measure of prosocial moral reasoning were significantly related to prosocial behavior. More specifically, hedonistic reasoning was negatively, and internalized reasoning was positively, related to peer ratings of prosocial behaviors. In addition, particularly for male adolescents, needs-oriented moral reasoning was positively related, and approval-oriented reasoning (which was frequently rejected by adolescents) was negatively related, to peer ratings of prosocial behaviors. These findings suggest that individuals who prefer other-oriented and sophisticated value-laden modes of moral reasoning are viewed by others as generous and helpful and that individuals who use self-focused and self-presentational types of reasoning are less likely to be viewed by others as generous and helpful. Perhaps more important, these findings generally were consistent with prior findings in other cultures, including samples of U.S. adolescents (see introduction).

There was some support for the notion that feminine gender role orientation was associated with prosocial moral reasoning. Adolescents who reported feminine characteristics were more likely to prefer internalized modes of prosocial moral reasoning and less likely to prefer approval-oriented reasoning (interestingly, as in Study 1, boys preferred approval-oriented reasoning more than did girls). In addition, boys (but not girls) who were feminine role oriented were more likely to prefer hedonistic reasoning. This latter finding may reflect the notion that hedonistic reasoning may be a viable, pragmatic way of thinking for male adolescents with counterstereotypic gender role preferences in a traditionally machismo-oriented society (there is some evidence that rejection of this traditional view may be occurring in Brazil; Hutz, Koller, \& Biaggio, 1992).

Somewhat unexpectedly, there was one significant relation between masculinity and prosocial moral reasoning (but only for boys). Boys (but not girls) who were masculine role oriented were less likely to be oriented toward the approval of others. This finding may reflect a belief by these male adolescents that actions based on gaining the approval of others (including parents and the general community) are considered less masculine. The present findings regarding the relations between gender role orientations and prosocial moral reasoning are not necessarily incompatible with Walker's (1991) or Baumrind's (1986) analyses of gender differences in moral reasoning or with McGraw and Bloomfield's (1987) analyses of the relations between gender role orientations and moral reasoning. Although one would expect both gender and gender role orientations to be multifaceted constructs and to be interrelated, each construct is considered uniquely multifaceted. For example, some researchers (see Huston, 1983) have argued that femininity and masculinity also tap into instrumentality, expressiveness, agency, and communion. Furthermore, whereas gender differences may be considered relatively fixed and rigid, gender role orientations presumably are more fluid and more sensitive to socialization experiences. In addition, there are distinct differences between Eisenberg's and Kohlberg's approaches not only with respect to the moral focus, but also in scoring methods (see Eisenberg, 1986); together these differences may account for different findings. It is clear that additional research is needed to further examine these issues.

\section{General Summary and Discussion}

Overall, the present findings extend our prior understanding of individuals' thinking of care-based, interpersonal-oriented social dilemmas. There was further evidence that proso-

Table 4. Correlations Between Prosocial Moral Reasoning and Femininity and Masculinity Within and Across Gender in Study 2

\begin{tabular}{llllllll}
\hline & \multicolumn{3}{c}{ Femininity } & & \multicolumn{3}{c}{ Masculinity } \\
\cline { 2 - 4 } \cline { 7 - 8 } Moral reasoning & Male & Female & Total & & Male & Female & Total \\
\hline Hedonistic & $.27^{* *}$ & -.15 & $-^{\mathrm{a}}$ & & .09 & -.06 & .02 \\
Approval & -.21 & -.17 & $.25^{* * *}$ & & $-.35^{* * *}$ & .08 & $-^{\mathrm{b}}$ \\
Needs-oriented & -.05 & .07 & .05 & & .21 & .11 & .15 \\
Stereotyped & -.08 & .14 & .07 & & .06 & -.01 & .01 \\
Internalized & .11 & .18 & $.18^{* *}$ & & .07 & -.14 & -.05 \\
\hline
\end{tabular}

Note: $n=55$ for male and $n=75$ for female adolescents.

${ }^{a}$ Gender $\times$ Hedonistic Moral Reasoning interaction was significant.

${ }^{\mathrm{b}}$ Gender $\times$ Approval-Oriented Moral Reasoning interaction was significant.

$* * p<.05$, two-tailed. $\quad * * * p<, 01$, two-tailed. 
cial moral reasoning is linked to prosocial behaviors in some Western cultures. Specifically, self-focused, hedonistic concerns were negatively associated with helpfulness and generosity, whereas abstract, other-oriented internalized concerns were positively associated with helpfulness and generosity. Furthermore, femininity was correlated with approval-oriented and internalized prosocial moral reasoning. Age and gender generally were related to prosocial moral reasoning in a theoretically expected manner and consistent with findings from U.S. samples. Moreover, the Portuguese version of the PROM appeared reliable and valid to use with Brazilian 5th to 10th graders (at least in a middle-class sample). Future studies designed to examine whether the correlates of prosocial moral reasoning are similar in other cultures may provide some insights about the underlying structures of moral reasoning.

\section{References}

Aiken, L. S., \& West, S. G. (1991). Multiple regression: Testing and interpreting interactions. Newbury Park, CA: Sage.

Akyel, B., \& Akyel, Y. (1991, June). Altruistic behavior and prosocial moral reasoning among Turkish high school students.Paper presented at the American Psychological Society Conference, Washington, DC.

Bar-Tal, D., Raviv, A., \& Lewis-Levin, T. (1980). The development of altruistic behavior: Empirical evidence. Developmental Psychology, 16, 516-524.

Baumrind, D. (1986). Sex differences in moral reasoning: Response to Walker's (1984) conclusion that there are none. Child Development, 57, 511-521.

Bem, S. L. (1974). The measurement of psychological androgyny. Journal of Consulting and Clinical Psychology, 42, 155-162.

Biaggio, A. M. B. (1976). A developmental study of moral judgment of Brazilian children and adolescents. Interamerican Journal of Psychology, 10, 71-78.

Boehnke, K., Silbereisen, R. K., Eisenberg, N., Reykowski, J., \& Palmonari, A. (1989). Developmental pattern of prosocial motivation: A cross-national study. Journal of Cross-Cultural Psychology, 20, 219-243.

Botenmpo, R., Lobel, S., \& Triandis, H. (1990). Compliance and value internalization in Brazil and the United States: Effects of allocentrism and anonymity. Journal of Cross-Cultural Psychology, 21, 200-213.

Carlo, G., Eisenberg, N., \& Knight, G. P. (1992). An objective measure of prosocial moral reasoning. Journal of Research on Adolescence, 2, 331-349.

Carlo, G., Knight, G. P., Eisenberg, N., \& Rotenberg, K. J. (1991). Cognitive processes and prosocial behaviors among children: The role of affective attributions and reconciliations. Developmental Psychology, 27, 456-461.

Colby, A., Kohlberg, L., Gibbs, J., \& Lieberman, M. (1983). A longitudinal study of moral judgment. Monographs of the Society for Research in Child Development, 48 ( Serial No. 200).

Eisenberg, N. (1986). Altruistic emotion, cognition and behavior. Hillsdale, NJ: Erlbaum.
Eisenberg, N., Boehnke, K., Schuler, P., \& Silbereisen, R. K. (1985). The development of prosocial behavior and cognitions in German children. Journal of Cross-Cultural Psychology, 16, 69-82.

Eisenberg, N., Carlo, G., Murphy, B., \& Van Court, P. (1995). Prosocial development in late adolescence: A longitudinal study. Child Development, 66, 1179-1197.

Eisenberg, N., Fabes, R., \& Shea, C. (1989). Gender differences in empathy and prosocial moral reasoning: Empirical investigations. In M. M.Brabeck (Ed.), Who cares? Theory, research, and educational implications of the ethic of care (pp. 127-143). New York: Praeger.

Eisenberg, N., Miller, P. A., Shell, R., McNalley, S., \& Shea, C. (1991). Prosocial development in adolescence: A longitudinal study. Developmental Psychology, 27, 849-857.

Eisenberg, N., \& Shell, R. (1986). The relation of prosocial moral judgment and behavior in children: The mediating role of cost. Personality and Social Psychology Bulletin, 12, 426-433.

Eisenberg, N., Shell, R., Pasternack, J., Lennon, R., Beller, R., \& Mathy, R. M. (1987). Prosocial development in middle childhood: A longitudinal study. Developmental Psychology, 23, 712-718.

Fuchs, I., Eisenberg, N., Hertz-Lazarowitz, R., \& Sharabany, R. (1986). Kibbutz, Israeli City, and American children's moral reasoning about prosocial moral conflicts. Merrill-Palmer Quarterly, 32, 37-50.

Gibbs, J. C., Arnold, K. D., Morgan, R. L., Schwartz, E. S., Gavaghan, M. P., \& Tappan, M. B. (1984). Construction and validation of a multiple-choice measure of moral reasoning. Child Development, 55, 527-536.

Gilligan, C. (1982). In a different voice: Psychological theory and women's development. Cambridge, MA: Harvard University.

Gilligan, C., \& Attanucci, J. (1988). Two moral orientations: Gender differences and similarities. Merrill-Palmer Quarterly, 34, 223-238.

Haidt, J., Koller, S. H., \& Dias, M. G. (1993). Affect, culture, and morality, or is it wrong to eat your dog?Journal of Personality and Social Psychology, 65, 613-628.

Hofstede, G. (1982). Dimensions of national cultures. In R.Rath, H. S.Asthana, D. Sinha. \& J. B. P. Sinha (Eds.)Diversity and unity in cross-cultural psychology (pp. 173-187). Lisse, The Netherlands: Swets \& Zeitlinger.

Huston, A. C. (1983). Sex-typing. In P. H.Mussen (Ed.), Handbook of child psychology: Vol. 4. Socialization, personality, and social development (pp. 387-467). New York: Wiley.

Hutz, C. S., De Conti, L., \& Vargas, S. (1993). Rules used by Brazilian students in systematic and nonsystematic reward allocation. Journal of Social Psychology, 134, 331-338.

Hutz, C., \& Koller, S. H. (1992). A mensura o de genero: uma readapta o do BSRI [The measurement of gender: A readaptation of the BSRI]. Psicologia: Reflexo e Cratica, 5, 15-21.

Hutz, C. S., Koller, S. H., \& Biaggio, A. M. (1992, August). Attitudes toward adultery, violence, and neglect in Brazilian men and women.Paper presented at the 100th annual convention of the American Psychological Association, Washington, DC.

Kurtines, W. M., \& Pimm, J. B. (1983). The moral development scale: A Piagetian measure of moral judgement. Educational and Psychological Measurement, 43, 89-105. 
Lang, J. (1988). Inside development in Latin America: A report from the Dominican Republic, Columbia, and Brazil. Chapel Hill: University of North Carolina Press.

McClelland, G. H., \& Judd, C. M. (1993). Statistical difficulties of detecting interactions and moderator effects. Psychological Bulletin, 114, 376-390.

McGraw, K. M., \& Bloomfield, J. (1987). Social influence on group moral decisions: The interactive effects of moral reasoning and sex role orientation. Journal of Personality and Social Psychology, 53, 1080-1087.

Pedhazur, E. J., \& Tetenbaum, T. J. (1979). Bem Sex Role Inventory: A theoretical and methodological critique. Journal of Personality and Social Psychology, 37, 996-1016.

Poppino, R. E. (1973). Brazil: The land and people (2nd ed.). New York: Oxford University Press.

Raviv, A., Bar-Tal, D., \& Lewis-Levin, T. (1980). Motivations for donation behavior by boys of three different ages. Child Development, 51, 610-613.
Rest, J. (1983). Morality. In P.Mussen (Series Ed.) \& J. H.Flavell \& E.Markman (Vol. Eds.), Handbook of child psychology: Vol. 3. Cognitive development (pp. 556-629). New York: Wiley.

Selman, R. (1980). The growth of interpersonal understanding. New York: Academic Press.

Taylor, M. C., \& Hall, J. A. (1982). Psychological androgyny: Theories, methods, and conclusions. Psychological Bulletin, 92, 347-366.

Tietjen, A. (1986). Prosocial reasoning among children and adults in a Papua New Guinea society. Developmental Psychology, 22, 861-868.

Underwood, B., \& Moore, B. (1982). Perspective-taking and altruism. Psychological Bulletin, 91, 143-173.

Walker, L. J. (1991). Sex differences in moral reasoning. In W. M.Kurtines \& J. L.Gewirtz (Eds.), Handbook of moral behavior and development: Vol. 2. Research (pp. 333-364). Hillsdale, NJ: Erlbaum. 